\title{
CATHODOLUMINESCENCE IN DOUBLE HETEROJUNCTION LASERS
}

\author{
P. HENOC, B. AKAMATSU and R.B. MARTINS $(1)$ \\ Centre National d'Etude des Télécommunications, CNET, 196, Avenue Henri \\ Ravera, F-92220 Bagneux, France
}

Resumé - La technique de Cathodoluminescence a été utilisée pour l'évaluation locale des propriétés de la couche active des lasers à double hétérostructure. L'analyse des profils et des spectres de cathodoluminescence, obtenus à partir des surfaces clivées, en fonction de la polarisation externe et du courant de faisceau, a permis une évaluation de la qualité des interfaces, de l'efficacité interne et du gain local de l'émission stimulée. Pour faciliter l'interprétation quantitative, une formule analytique de la fonction de génération, dérivée de la simulation de Monte-Carlo, a été utilisée.

Abstract - The cathodoluminescence mode of the Scanning Electron Microscope is used to evaluate local properties of the active layer of double-heterojunction lasers. The analysis of the Cathodoluminescence line-scans and spectra from cleaved laser surfaces, as a function of the external bias and of the incident beam current $t_{r}$ give information about the interface quality, the internal efficiency and the local gain of stimulated emission. In order to make a quantitative interpretation of our experimental measurements, an analytical form of the tridimensional generation function, derived from a Monte-Carlo trajectory simulation, is used.

\section{Introduction}

The early progress in optoelectronic materials has been accompanied by an enhancement of the number of characterization techniques of these materials. Among these techniques, the Cathodoluminescence (CL) has been used as a very important tool for the study of direct band-gap semiconducting materials/1,2/, often used in optoelectronics.

Usually, the structure of an optoelectronic device includes several epitaxial layers with different chemical compositions (heterostructures). To evaluate the properties of these epilayers and heterointerfaces, many authors are working in isotype double-heterostructures (namely, structures with different chemical compositions but identically doped) using CL or photoluminescence measurements $13,4,5 \%$. In these works, the quantum efficiency of the layers, the recombination velocity of the interfaces and some energy band properties have been evaluated. In these experiments problems due to the electrical junction (in an actual laser device, related to doping differences) or due to the final device fabrication can not be observed. Consequently, it seems to be interesting to investigate the CL possibilities as a characterization technique for double-heterojunction lasers (DH-lasers).

For this purpose, we have analysed the CL line-scan signal from cleaved laser surfaces. The quality and spatial homogeneity of GaAlAs/GaAs and InGaAsP/InP DH-lasers have been observed with spectroscopically resolved $\mathrm{CI}$.

\section{General considerations}

\section{II.a. Cathodoluminescence.}

The scanning electron microscope (SEM) CL-mode can be used for high spatially resolved observation of the local properties of semiconducting materials $/ 1,2,3 /$. The recent technological developments of the light collection systems and of the cold stages, have enhanced the possibilities of this technique. Spectral CL is used to improve the available information on the different physical processes that can occur in the sample when it is bombarded by an electron beam. It is often interesting to work at low temperature, where excitonic emissions predominate, e.g.: free-exciton, bound-exciton and donor-acceptor transitions; the corresponding CL signals can be used, for example, to observe quantum-well homogeneity /6/

(1) Supported by CNPG and FUNCAMP from Brazil 
or to identify local deep-level transitions $17 /$. When the $\mathrm{CL}$ signal is weak, the low-temperature condition is also often suitable, since it enhances the CL efficiency of the sample 181 .

At room temperature, band-to-band and deep-level transitions predominate within the $\mathrm{CL}$ signal Room-temperature CL measurements have been used to evaluate several semiconductor parameters. In the case of a homogeneous crystal or a thick layer (semi-infinite semiconductor), the variation of the $C L$ intensity with the electron beam voltage, when compared to the theoretical calculations, makes it possible to evaluate the minority carrier diffusion length, the surface recombination velocity and the optical absorption coefficient of the sample $/ 9,10 /$. The theoretical approaches are based on a diffusion equation with suitable boundary conditions /11/ and a generation function (or depth-dose distribution) of excess carriers.

\section{II.b.Generation function}

As many authors have pointed out $/ 12,13 /$, the choice of the generation function is very important to obtain a good theoretical approach and consequently, a good accuracy for the SEM-CL or SEM-EBIC (Electron Beam Induced Current) measurements of semiconductor parameters. Several generation functions are proposed and studied by many authors: a polynomial form by Everhart and Hoff /14/, a Gaussian function by Wu and Wittry /15/, a modified Gaussian function by Donolato /16/ and by Oelgart and Scholz /17/, a double Gaussian form by Konnikov /18/ and a Gaussian function (Donolato) plus the Everhart and Hoff polynomial by Closas /19/.

To interpret qualitatively our experimental measurements, we are led to use a very fine generation function, since the dimensions of the studied layers are small in comparison to the generation volume. We have found an analytical expression for the generation function including three Gaussian terms /20/. These Gaussian functions have their variances correlated to the Gruin range /21/. The amplitudes of each Gaussian term is also a Gaussian function of the depth. The first Gaussian predominates far from the impact point : it represents the backscattered electrons contribution. The second one is correlated to low angle inelastic scattering: it is important near the impact point. The third one is due to the primary electrons themselves, and it has to be taken into account only for depths greater than the inelastic mean free path. This expression gives a good agreement with Monte Carlo calculations for a mean atomic number $Z=30$ (which is the case for the majority of III-V semiconducting compounds) at every primary energy from 10 to $60 \mathrm{keV}$.

\section{II.c. Double-heterojunction lasers}

The materials commonly used for DH-laser fabrication are the III-V compounds /22/. The choice of these materials is related to several advantages. For example, for a good epitaxial growth, it is possible to choose from a large range of alloys composition with aifferent energy-gaps and lattice parameters nearly equal to the substrate ones ( the substrates commonly used are GaAs or InP wafers).

In figure 1 we show a scheme of a DH-laser structure. During the device operation, the radiative recombination is limited to a thin layer, named the active layer. This active layer is sandwiched between two layers of larger gap and of lower refractive index. These layers play the role of electrical and optical confinement and so, it is possible to obtain the high injection level necessary to produce the stimulated emission 123 . The resonant cavity of the laser is obtained by two cleaved facets in the (110) direction. Usually, the epitaxial growth is initiated by a buffer layer to eliminate the influence of the substrate defects on the quality of the next epitaxial layers. Following the double heterojunction structure, a contact layer with a high doping level is grown on the top of the crystal After the growth, the crystal is submitted to several technological processes necessary for the device fabrication, e.g.: thinning, cleavage, film evaporation, alloying and so on /22/.

The device reliability and performance are related to the quality and control of these processes. Some of the characteristics that can be modified during these steps are, for example: the efficiency of the active layer, the trap densities at the heterointerfaces, the current leakage, regions with local absorptions or non-radiative recombination sites, the thermal conductivity of the layers, the quality of the mirror facets, etc. 
The aim of this work is to study the possibilities of the CL technique in the evaluation of the properties listed above and of the influence of each technological process on these properties. This study may be useful to improve the quality and the reliability of the final device.

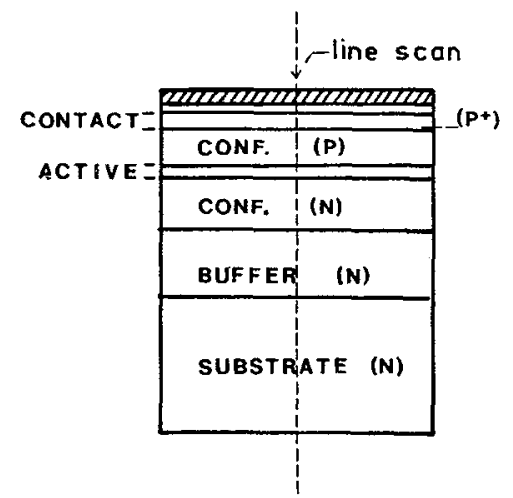

FIGURE 1 : Schema of a typical DH-Lasex for optical telecommunications. The line scan corresponding to the experimental procedure is shown (see text).

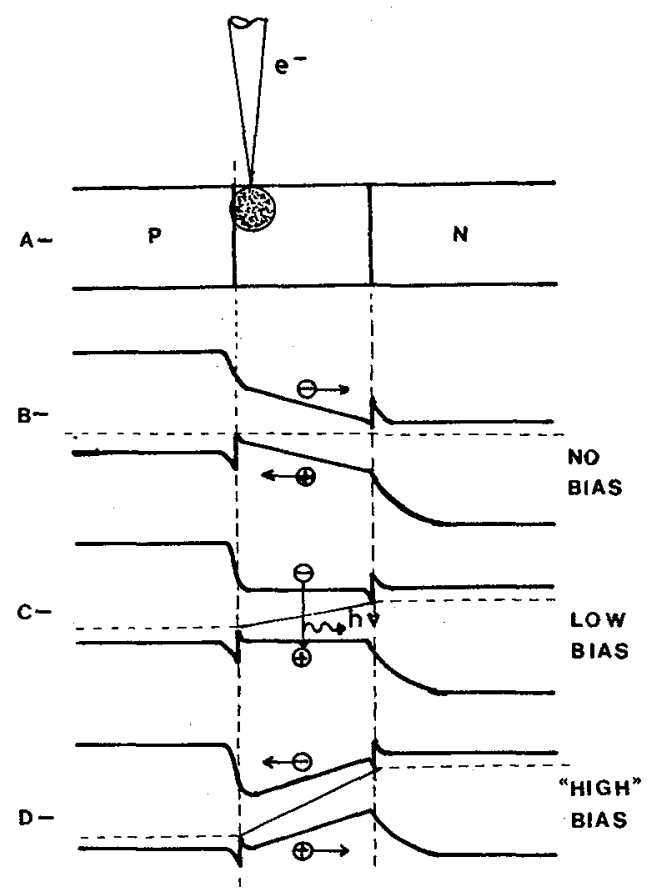

FIGURE 2 : Influence of the forward bias on the energy bands of a heterojunction : a) scheme of the semiconductor structure; b) without external bias; c) with a low bias or an internal polarization; d) with a high forward bias. 


\section{Experimental procedure}

In our experiments, the CI signal is obtained when the electron beam scans on a cleaved surface perpendicularly to the junction plane (see fig. 1). This signal is compared to the EBIC and to the Electroluminescence (EL) signals for a good interpretation of the experimental results.

Many studjes using the CI and EBIC line-scans across a junction have been published, specially for GaP diodes $124,25,26,27 /$. As we can observe in these measurements, the $C L$ signal is minimum when the electron beam is localized in the depletion region. To observe successfully the active layer emission of a DH-laser it is then necessary to decrease the depletion zone width to reduce the effect of carrier separation. This effect can be avoided by the application of an external bias /28/ or by the creation of an internal polarization due to a local accumulation of carriers generated by the electron beam itself.

If one side of the DH-laser sample is connected to the ground and if the other one is not electrically connected, the excess carriers can drift to the disconnected region and reduce the fixed charge density therein (figure 2b). After a sufficient concentration of excess carriers, we can obtain an equilibrium regime when some electron-hole pairs generated may be confined in the active layer and so are able to recombine radiatively (figure 2c). This effect which reduces the "EBIC" and enhances the CL is in competition with the non-radiative recombination process on traps at the heterointerfaces. So, for a given trap density we can obtain a different equilibrium regime for each excitation condition. It is interesting to note that, in this condition, by increasing the excess carrier density, we can attempt the regime of the stimulated emission in the active layer.

Our measurements are made on the SEM JEOL-JSM 840 where the electron source is a LaB6 crystal Figure 3 is a scheme of the experimental configuration. The CI signal is collected by an elliptical mirror and guided to the spectrometer by a light pipe. The Germanium avalanche-photodiode signal is amplified and recorded using a mini-computex (or a recorder). The signal-to-noise ratio is improved by a beam-blanking device and a lock-in amplifier.

The electron beam current is measured using a Faraday cup device and a picoammeter. A low-noise DC power supply is used to bias the sample, and the injected current throughout the sample is controlled during the experiment. The use of the beam blanking device and the lock-in amplifier allows the measurement of the pulsed CL and EBIC signals separated from the Electroluminescence ( $\dot{E} L)$ signal and the injected continuous current (similarly to ref. 28) .

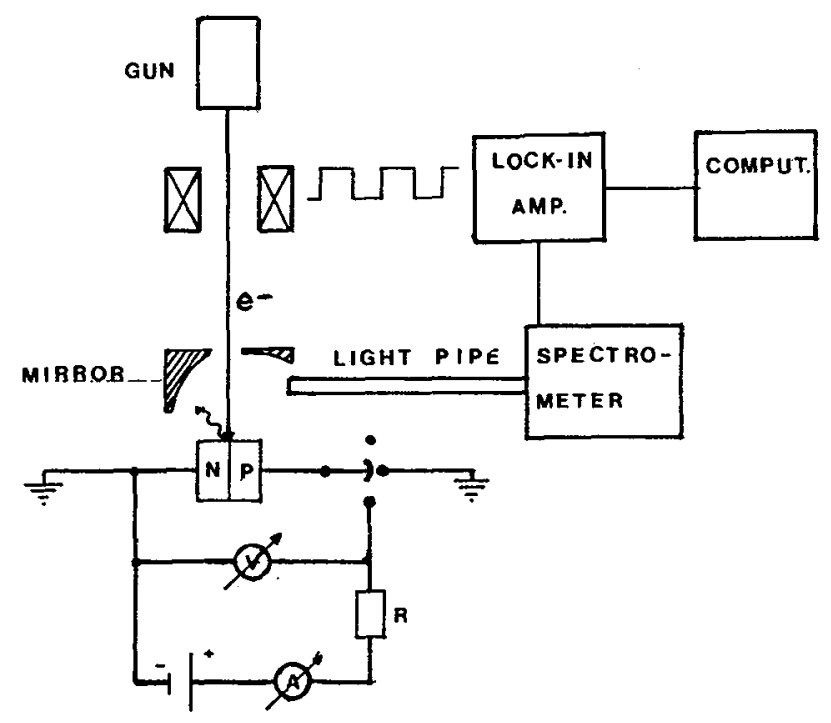

FIGURE 3 : Schema of the experimental configuration used for the SEM characterization of the DH-Lasers. 
The samples analysed are DH-lasers grown by different epitaxial techniques and with different material compositions, namely:

i)GaAlAs/GaAs DH-lasers (three samples): grown by metal-organic chemical vapour deposition (MOCVD) (\#1) or by molecular beam epitaxy (MBE) (\#2 and \#3). The structures of these as-grown samples are basically the same. A difference occurs in the dopant type because there are some limitations for each technique: the MBE growth uses beryllium as the acceptor and zinc is used in the place of beryllium in the case of the MOCVD growth. For donor doping, silicon is used in both techniques. The typical characteristics of these samples are listed bellow:

\begin{tabular}{||c|c|c|c|c|c|}
\hline Layer & Composition & Thickness $(\mu \mathrm{m})$ & $\begin{array}{c}\text { Doping }(\mathrm{cm}-3) \\
(\# 1, \# 3)\end{array}$ & $\begin{array}{c}\text { Energy gap } \\
\text { (eV) }\end{array}$ \\
\hline Buffer & GaAs & 3.0 & $8 \times 10^{17}$ & $\mathrm{~N}$ & 1.42 \\
\hline Confin. & GaAlAs & 1.0 & $8 \times 10^{17}$ & $\mathrm{~N}$ & 1.79 \\
\hline Active & GaAs & 0.2 & undoped & & 1.42 \\
\hline Confin. & GaAlAs & 1.0 & $8 \times 10^{17}$ & $\mathrm{P}$ & 1.79 \\
\hline
\end{tabular}

ii) InP/InGaAsP DH-lasers (two samples): grown by liquid phase epitaxy (LPE). In this case, cadmium is used as acceptor and tin as donor in both samples. It is important to note three differences between these samples: the sample \#4 is an as-grown crystal whereas the sample \#5 is a final device. Furthermore, the chemical compositions and thicknesses of the two active layers are different. The typical characteristics of these samples are:

\begin{tabular}{|c|c|c|c|c|}
\hline Layer & Composition & Thickness $(\mu \mathrm{m})$ & Doping( $\left.\mathrm{cm}^{-3}\right)$ & $\begin{array}{c}\text { Energy gap } \\
(\mathrm{eV})\end{array}$ \\
\hline buffer & InP (N) & 4.0 & $2 \times 10^{18}$ & 1.32 \\
\hline active & InGaAsP & $0.4(\# 4)$ & $-10^{15}$ & 0.95 \\
& & $0.15(\# 5)$ & $-10^{15}$ & 0.83 \\
\hline confin. & InP (P) & 2.0 & $2 \times 10^{18}$ & 1.32 \\
\hline
\end{tabular}

All the as-grown samples were thinned to approximately 200 microns, cleaved and then immediately put into the SEM chamber, to avoid too much surface contamination.

\section{Results}

In figures 4 and 5 the filtered $C L$ intensity is plotted along a line-scan perpendicular to the junction plane on the cleaved facet (110) of the GaAlAs/GaAs DH-lasers (\#1, \#2 and \#3). In this work, the applied accelerating voltage for the electron beam is always $10 \mathrm{kV}$.

In all cases, for the active layer characteristic emission ( $\lambda \oplus .89$ micron), the CL intensity grows quasi-monotonously when the beam scans from the surface to the buffer layer. The only accidents observed are located near the buffer-substrate interface and they are characteristic of defects introduced by the substrate itself or by the substrate chemical preparation before growth.

We can also observe that the emissions from the GaAlAs layers $(\lambda=0.68$ micron) are minima near the active region. The maximum of these signals are characteristic of the internal efficiency of each confinement layer, of its minority carrier diffusion length and of the surface and interface recombination velocities. It is interesting to note that, for these samples, the CL intensity is always low for the Be-doped MBE layer. This result is the same for the samples \#2 and \#3 which means that the internal efficiency of the Be-doped layer is weak wherever it is grown: after the buffer layer (\#2) or after the active layer (\#3); if we compare the CL intensity from the different buffer layers, we can see that the CL signal of the MBE Si-doped buffer layer is, at least, six times stronger than the CL signal of the Be-doped ones. It seems that the low efficiency of the Be-doped layers is due basically to the small ionic radius of the beryllium atom which enhances the probability for it to lie at an interstitial site. 
For the Si-doped MOCVD layers we detect a non intentional variation of the aluminium concentration giving very thin layers (10 nanometers) with different energy gaps; each of these layers can play the same role as a quantum-well and they are randomly distributed within the confinement layer.

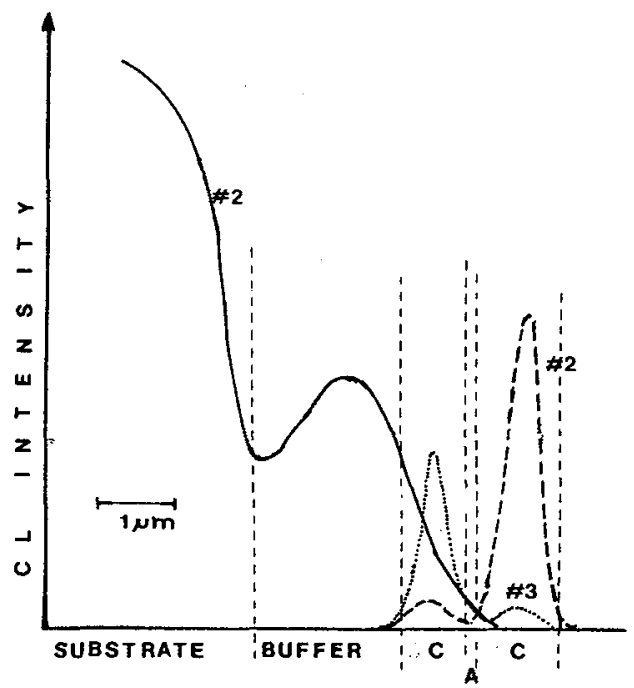

FIGURES 4 : $C L$ line-scans of the MBE DH-lasers. The normal line represents the GaAs signal. The dotted line is the CL signal from the GaAlAs layers of the sample with the P-type layer in the surface (\#3) and the discontinuous line represents the GaAlAs CL from the sample with the N-type layer in the surface (\#2).

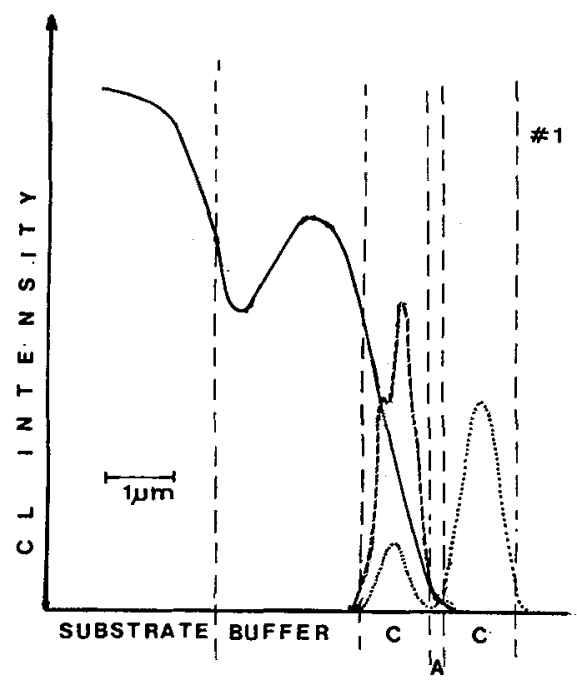

FIGURE 5 : CL line-scans of the MOCVD DH-lasers. The normal line represents the CL signal filtered for the GaAs emission. The dotted line represents the CL signal filtered for the expected GaAlAs emission (0.68 micron) and the discontinuous line is the $\mathrm{CL}$ signal for the anomalous emission ( 0.75 micron) occured in the Si-doped layer.

As we can observe, in these measurements it is possible to obtain some information about the confinement layer and the buffer layer, but it is very difficult to analyse the active layer, since the CL signal of the buffer layer (which have an energy gap nearly equal to the active layer ones) always dominate the CI line-scan (when the electron beam is on the active layer, the generation volume overlaps the confinement layer so the minority carriers can diffuse to the buffer layer and recombine therein). In the active region, the carrier drift reduces the radiative recombination probability, and we do not observe any important difference on the CL line-scans of these samples, even at very high excitation conditions. In this case, it is preferable to use the EBIC measurements to evaluate the collection efficiency of the diode.

Following the same experimental conditions, we have made CI line-scans on the as-grown LPE InGaAsP DH-laser (\#4). At relatively low-injection conditions (Ib<20nA), we can observe, similarly to the GaAlAs samples, the strong signal of the buffer layer and the signal of the confinement $\mathrm{N}$-doped layer. At the wavelength corresponding the active layer emission ( $\lambda=$ 1.3 micron), we note again a monotonous progression of the CL-signal when the beam scans from the surface to the substrate. After verification, it is demonstrated that the enhancement of the CI line-scan in this wavelength is caused by deep-level transitions in the InP buffer layer.

In figure 6 , we show the variation of the CI line-scan with the electron beam current. We can observe that for a beam current of $20 \mathrm{nA}$ a little "peak" appears in the region of the active layer. It means that the auto-polarization effect begins to enhance the radiative recombination . 
This regime corresponds to a local saturation of the traps at the heterointerfaces and it is possible to map the density of traps along the junction plane, by measuring the different values of the beam current necessary to promove this regime. Using this "threshold" value and calculating the correspondent density of pairs generated into the active layer, we can estimate locally the product $N \times \%_{0}$ where $N$ is the trap density, $\tau_{n r}$ and $\tau_{r}$ the non-radiative and the radiative lifetimes respectively. This sample was very homogeneous and the average value obtained is $N=3.0 \times 10^{13} \tau_{\mathrm{nr}} \mathrm{cm}^{-2}$. If we consider the ratio $\tau_{\mathrm{nr}} / \tau_{r} \sim 0.1$, we obtain an estimated value for $N$ in the order of $10^{12} \mathrm{~cm}^{-2}$.

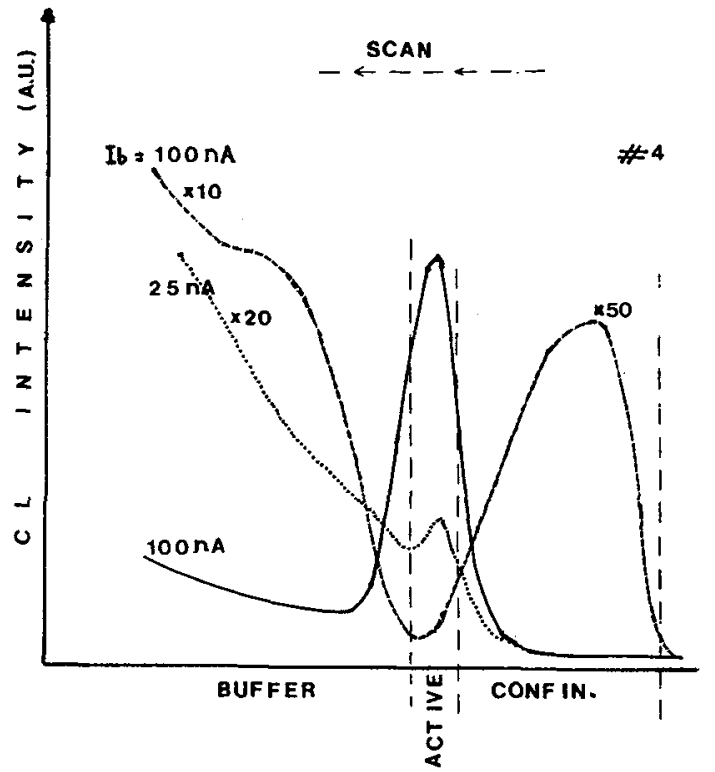

FIGURE 6 : Evolution of the CL line-scans from the LPE DH-laser (\#4) with the beam current. The discontinuous line is the CL signal for the InP (0.93 micron) emission with $I_{b}=100 \mathrm{nA}$; the dotted and the normal lines correspond to the 1.3 micron emission for two different values of the beam current.

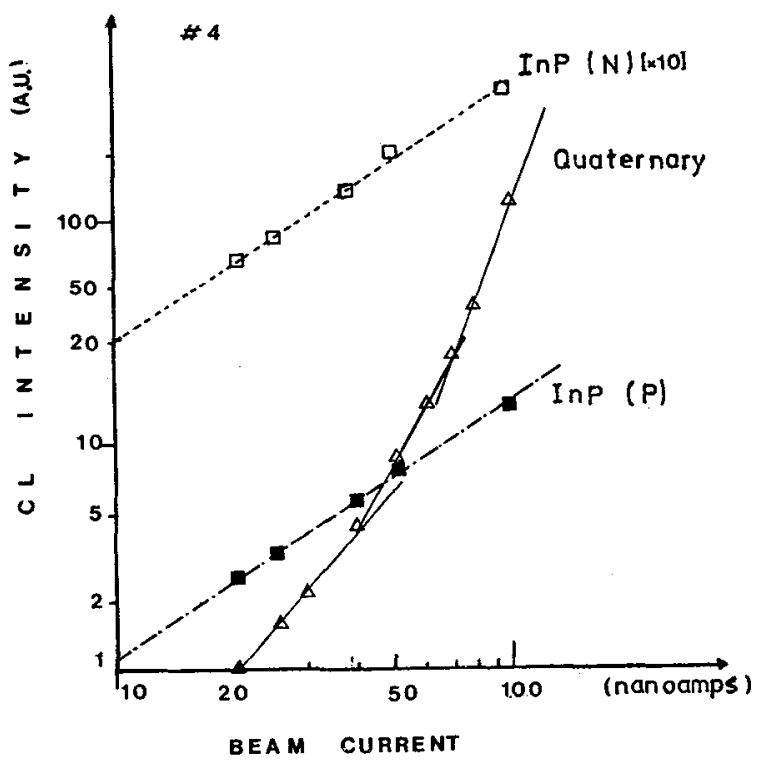

FIGURE 7 : CL intensity as a function of the beam current from the quaternary active layer emission (1.3 micron). The signals of the two confinement layers (square symbols) are showed for comparison purposes.

Still in figure 6, for a beam current of $100 \mathrm{nA}$, we observe that the CL intensity of the active layer increases strongly. It means that a regime of stimulated emission is obtained.

The evolution of the CL intensity as a function of the beam current is shown in figure 7. For the active layer characteristic emission, in the range of $20 \mathrm{nA}$ and approximately $50 \mathrm{nA}$, we observe a quadratic regime. In this steady-state condition, a part of the excess carriers recombines non-radiatively, another one drifts and reduces the fixed charge density; so, the probability of radiative recombination in the active layer is enhanced. Furthermore, calculating the excess carriers density, we can observe that we are in the regime of bimolecular recombination which can explain the quadratic dependence. For these excitation conditions, the emission of the active layer becomes stronger than the deep-level ones and we are able to characterize the active layer itself. For example, a map of the local CL efficiency can be made.

After $50 \mathrm{nA}$, the CL intensity increases very rapidly (slope>5). It corresponds to a regime of stimulated emission. This threshold current for the local stimulated emission gives a calculated carrier density of $6 \times 10^{17} \mathrm{~cm}^{-3}$, by using our generation function and a given $\tau$ (total minority carriers lifetime) of $10^{-9}$ seconds. In this condition, it is possible to obtain a map of the optical gain, without the influence of the facet mirrors and of the ohmic contacts. In order to verify our assumption of a stimulated emission, we have also measured the variation of the CL signal from the InP layers (where the stimulated emisssion is not expected) as a function of the beam current. As we show in figure 7, even at $100 \mathrm{nA}$, the quasi-linear regime is maintained for these layers. 
In order to control the effective stimulated emission phenomena, we have observed the evolution of the CL spectra with the beam current. In figure 8a we show the active layer CL spectra at a low-excitation condition. By increasing the beam current, several peaks appear on the spectra (fig. $\mathbf{8 b}$ and $\mathbf{8 c}$ ). They could be related to chemical composition variations in the excited region. This observation can be correlated, for instance, to the demiscious phenomena observed in this alloy when it is grown by LPE $129 /$, and this could be spectrally verified only in the conditions of stimulated emission.

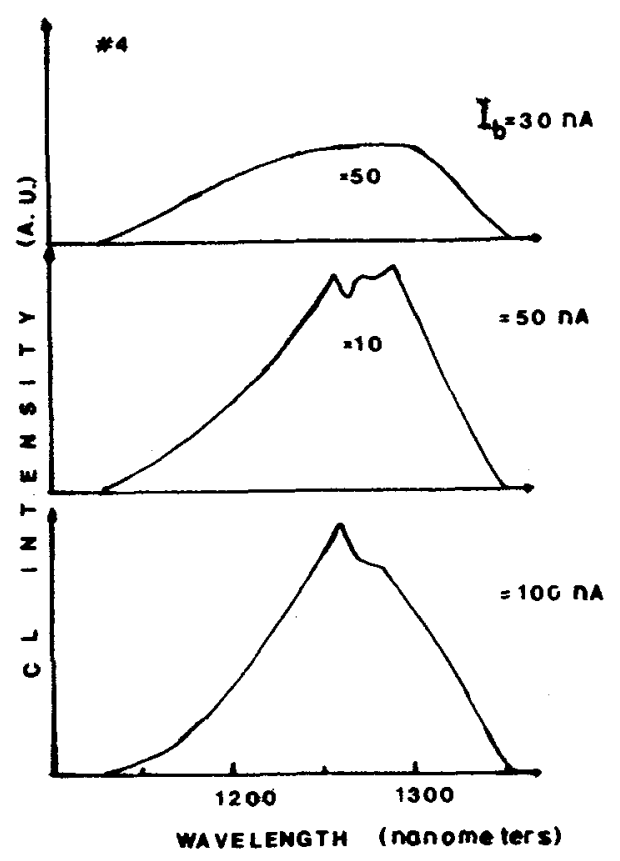

FIGURE 8 : Evolution of the CI spectra from the quaternary active layer as a function of the electron-beam current.

The next results were obtained for the final laser device. For this sample, with one side disconnected, the CL signal of the active layer was only observed at high excitation conditions (500 $\mathrm{nA} ; 10 \mathrm{kV})$.

When the trap density at the heterointerfaces is sufficiently high to avoid the auto-polarization effect, even at high excitation conditions for the SEM, it is still possible to observe the CL signal of the active layer by applying an external forward bias at the diode (see figure 2c). Similarly, a part of the excess carriers generated by the electron beam can be confined into the active layer and recombines radiatively, when the sample is sufficiently externally biased.

In figure 9 we have plotted versus the external bias: the diode injected current, the CL signal of the active layer, the EBIC and the EL signal for a given excitation condition (namely $100 \mathrm{nA}, 10 \mathrm{keV})$.

In this figure, we can observe that the $C L$ signal from the active layer appears with a very low bias $(0.3 \mathrm{~V})$, while the EBIC is still important. In this condition we can map by CL, the internal efficiency of the active layer and by EBIC, the homogeneity of the electrical junction. The competition between these two phenomena is evident in this figure. 


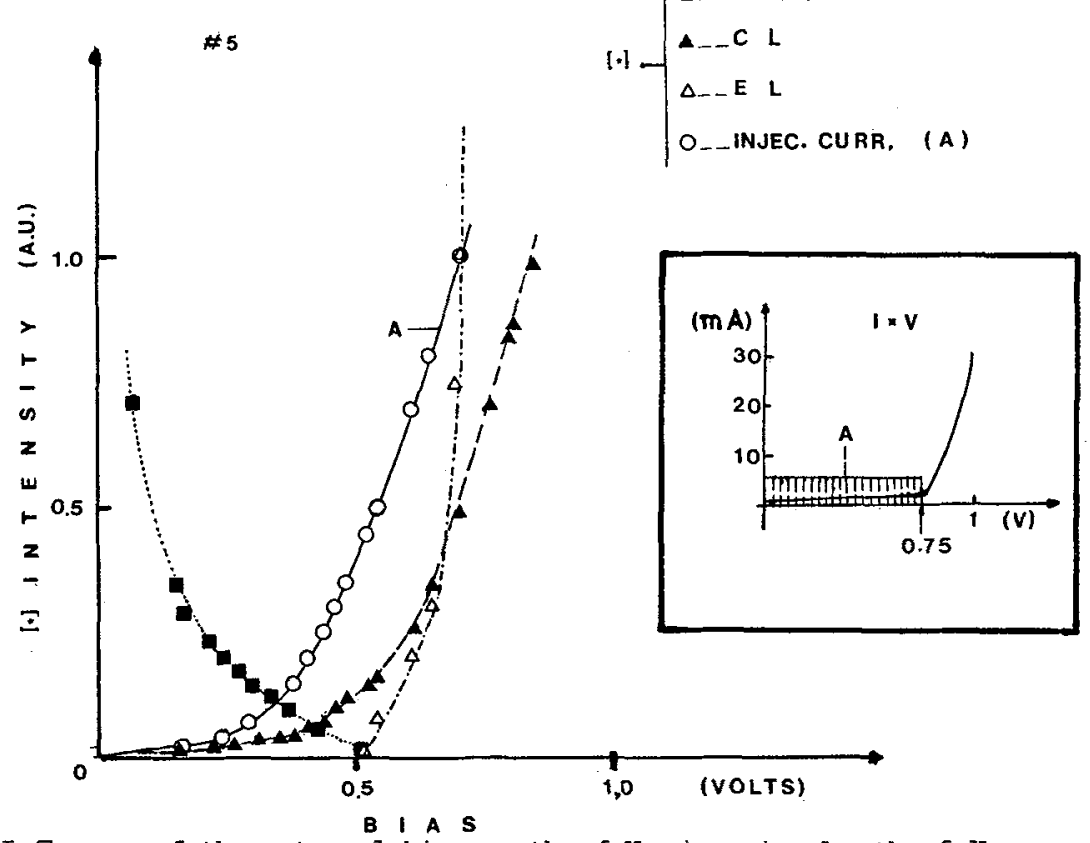

FIGURE 9 : Influence of the external bias on the following signals: the full squares represent the EBIC signal; the full triangles correspond to the CL signal of the active layer (1.5 micron), we can observe the "competition" between these two signals. The electroluminescence and the injected current in the sample are also represented. To avoid some mistakes in the interpretation of these curves, the inset shows the direct I-V characteristic curve of this diode (all our measurements have been made in the non-ohmic region of the diode, cf. A).

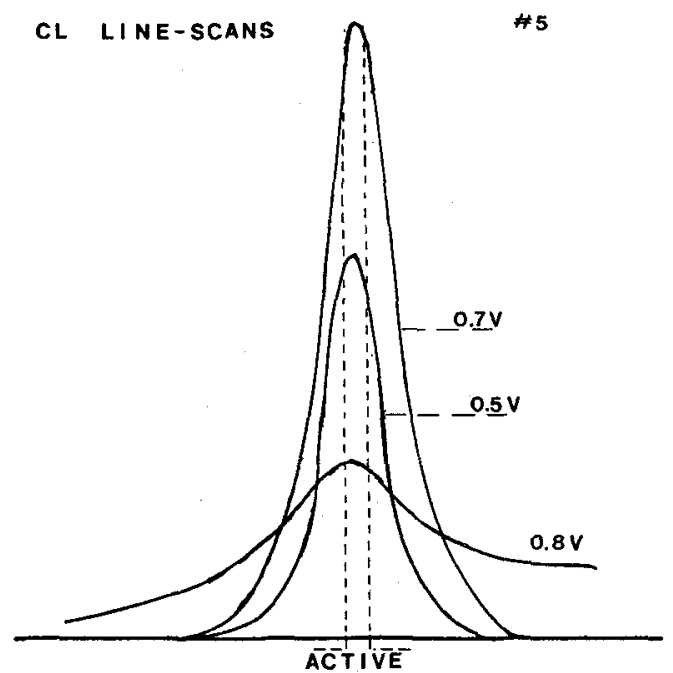

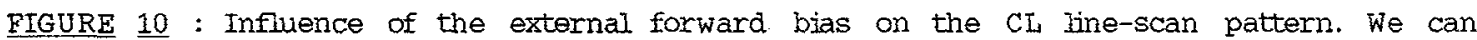
observe the decreasing and the broadening of the line-scan as the forward bias is increased.

When the EBIC is negligible (bias $>0.5 \mathrm{~V}$ ), the major part of the excess carriers recombine radiatively and the CL signal. grows linearly with the direct injected current. In this regime it is possible to obtain a map of the carriers injected by the external bias, that means, the current confinement of the device. This regime is also suitable to characterize, for instance, low efficient diodes (for example, degraded lasers), since the injected current plays the role of an internal amplifier for the CI signal Unfortunately, this is not the case for high forward bies. With bias greater than $0.5 \mathrm{~V}$, the EL signal increases much more rapidiy than the CL 
signal. It means that the radiative recombination transitions results more from the majority carriers injected at each side of the junction than from the electron-hole pairs generated within the active layer. Furthermore, at higher forward bias, when the externally applied electric field is greater than the energy gap $\left(V \approx E_{g} / e\right)$, the excess carriers generated by the electron beam can drift outside of the active layer (see figure 2d). So, they will drift in the same way as the injected carriers and contribute to the EL signal Consequently, the CL signal decreases and remains independent of the electron beam position on the sample. Obviously, this situation is not suitable for SEM observations. Figure 10 illustrates this phenomena.

\section{Conclusion}

To analyse a DH-laser we are mainly interested in the study of its active layer, but the presence of a space charge region (heterojunction) reduces considerably the CL signal from this layer. In this work, we show how the polarization effects may be used to enhance the CL signal from the active layer. The analysis of the CL signal from a cleaved surface was used to obtain important information about the quality and homogeneity of the epilayers and heterointerfaces.

In the goal of reducing the electrical field in the active region, we study the effect of the auto-polarization on an as-grown DH-laser and the regime of stimulated emission is obtained. In the case of a final device, an external forward bias is applied. In this condition, it is possible to observe the CI and EBIC signals simultaneously with the EI signal An analysis of the dependence of these signals with the applied voltage bias is very useful to interpret the measurements and to study several properties of the device (the current confinement, for example). Furthermore, the forward bias may be used to increase the CL intensity, which is very interesting to study (at room temperature) devices with low $C I_{1}$ efficiency.

In conclusion, our experiments show that the CL may be used as a powerfull technique for DH-laser characterization. As we analyse the CI signal from a cleaved facet (usually, in the (110) direction), we are able to observe, in the same experimental conditions, an as-grown or a final laser. This is important to evaluate the actual influence of each technological process in the characteristics of the final device. Furthermore, the heterojunction may be considered as a "defect" region, because it is a region with a local electrical field and a local minority carriers lifetime different from the "bulk" material The technique used in this work can give some contributions to a best comprehension of the influence of an "anomalous" region in the diffusion and the generation of excess carriers in a semiconducting material

Acknowledgements: The authors wish to thank greatfully R. Charil, R. Azoulay, G. Chaminant and $F$. Alexandre for having provide the samples used for this work.

\section{REFERENCES}

/1/ YACOBI B. G. and HOLT D.B., J. Appl Phys. 59 (1986) R1-R24

/2/ SPIVAK G. V., PETROV V. I. and ANTOSHIN M. K., Sov. Phys. Usp. 29 (1986) 364-380

/3/ LIANG-HUI CHEN, MATTOS J. V. C., PRINCE F. C. and PATEL N. B., Solid State Comm. 53

(1985) $441-444$

/4/ SERMAGE B., PEREIRA M. F., ALEXANDRE F., BEEREINS J., AZOULAY R., TALLOI C., JEAN-IOUIS A.M. and MEICHENIN D., Journal de Physique C5, 48 (1987) 135-138

15/ KONNIKOV S. G., SOLOV'EV V. A., UMANSKI V. E., CHUDINOV A. V. and KHUSAINOV A. A., Sov. Phys. Semicond. 20 (1986) 661-664

16/ BIMBERG D. - private communication

17/ PAPADOPOULO A. C., BRESSE J. F. and PRASEUTH J. P., J. Appl Phys. 62 (1987) 4174-4177

18/ JONES G. A. C., NAG B. R, and GOPINATH A., in 6th Ann. SEM Symp., IITRI, Chicago (1973) 309-316

19/ MIKHEEV N. N., Sov. Phys. Semicond. 21 (1987) 226-227

110/ KOCH F. and OELGART G., phys. stat. sol (a) 104 (1987) 931-939

/11/ van ROOSBROECK W., J. Appl Phys. 26 (1955) 380-391

/12/ FIDDICKE J. and OELGART G., phys. stat sol (a) 87 (1985) 383-389

113/ KONNIKOV S. G., SOLOV'EV V. A., UMANSKI V. E., KHUSATNOV A. A., CHISTYAKOV V. M. and YASSIEVICH I. N., Sov. Phys. Semicond. 21 (1987) 997-1000

/14/ EVERHART T. E. and HOFF P. H.; J. Appl Phys. 42 (1971) 5837-5846 
/15/ WU C. J. and WITTRY D. B., J. Appl Phys. 49 (1978) 2827-2836

/16/ DONOLATO C., phys. stat. sol. (a) 65 (1981) 649-658

/17/ OELGART G. and SCHOLZ H., phys. stat. sol. (a) 75 (1983) 547-553

18/ KONNIKOV S. G., SOLOV'EV V. A., UMANSKI V. E. and CHYSTIAKOV V. M., SOV.PhYs. Semicond. 21 (1987) 1229-1231

119/ CLOSAS L. and FERNANDEZ RUBIO J., phys. stat. sol (a) 105 (1988) 541-548

120/ HENOC P. and AKAMATSU B., to be published

/21/ GRÜN A. E., Z. Naturf. 12a, (1957) 89

122) see, for example: KRESSEL H. and BUTLER J. K. in "Semiconductor Lasers and Heterojunctions LEDs" Ac. Press New York 1977 and KRESSEL H. in "Semiconductor Lasers" in: Hilsum C. "Handbook of Semiconductors; vol 4; North-Holland Publ 1981.

123/ PILKUHN M. H., Journal of Luminescence 7 (1973) 269-283

124/ STRINGFELLOW G.B. and KERPS D., Solid-State Electr. 18 (1975) 1019

125/ OELGART G., FIDDICKE J. and REULLE R., phys. stat. sol (a) 66 (1981) 283-292

/26/ D. B. Holt et al; phys. stat. sol (a) 20, 459 (1973)

127/ BARINOVA E. YU., VISHNEVSKAYA B. I., GOLUBEV YU. A., KOVYREVA N. I., KOGAN L. M., PETROV V. I. and YUNOVICH A. E., Sov. Phys. Semicond. 13 (1979) 282-287

/28/ HAEFNER H., MORAWETZ H. and OELGART G., phys. sta. sol (a) 63 (1981) 495-500

129/ HENOC P., IZRAEL A., QUILLEC M. and LAUNOIS A., Appl PhYs. Lett 40 (1982) 963 